\title{
Loss of nucleolar-organizer regions during polyploid evolution in Scilla autumnalis
}

\author{
H. E. VAUGHAN', M. JAMILENA², C. RUIZ REJÓN², J. S. PARKER ${ }^{1 *}$ \& M. A. GARRIDO- \\ RAMOS ${ }^{2}$ \\ 'School of Biological Sciences, Queen Mary and Westfield College, University of London, Mile End Road, London E1 \\ 4NS, UK and 2 Departamento de Genética, Facultad de Ciencias, Universidad de Granada, 18071 Granada, Spain
}

\begin{abstract}
The organization and behaviour of nucleolar-organizer regions (NOR) of Scilla autumnalis were studied in two diploid races, their allotetraploid and autoallohexaploid derivatives and hybrids between them. The diploids of genome constitution $A A$ and $B^{7} B^{7}$ each have a pair of nucleolarorganizer chromosomes while the polyploids $A A B^{7} B^{7}$ and $A A B^{7} B^{7} B^{7} B^{7}$ only carry visible organizers on the $B^{7}$ chromosomes. In situ hybridization using ribosomal probe pTA71 was used to localize ribosomal cistrons. In polyploids the sites of hybridization are limited to the $B^{7}$-derived nucleolar-organizer chromosomes and ribosomal cistrons have thus been deleted from the $\mathrm{A}$ genome. A NOR-associated C-band has also been deleted.
\end{abstract}

Keywords: allopolyploids, in situ hybridization, nucleolar dominance, nucleolar-organizers

\section{Introduction}

In polyploids there are frequently fewer nucleolarorganizer regions (NORs) visible in the chromosome complement than would be expected from the sum of the diploid genomes. Tetraploids, for example, may show only a single pair of NOR chromosomes, as in Scilla scilloides (Araki, 1985) or Milium montianum (Bennett \& Thomas, 1991). Clearly a reduction in the number or expression of NORs must have occurred at the time of, or subsequent to, polyploidization. In some interspecific hybrids of diploid and polyploid plants the NORs derived from one parent may not be expressed as visible secondary constrictions in the chromosome complement. This is the phenomenon of nucleolar dominance or amphiplasty (Navashin, 1934; Flavell, 1989). The inactive NORs, however, can be reactivated in backcrosses, demonstrating that suppression is not a permanent change in the karyotype. Nucleolar dominance, therefore, could underlie the observations of reduced numbers of NORs in the genomes of polyploids. An alternative explanation is that deletion of all or some of the ribosomal cistrons or controlling elements which comprise the NOR region may have occurred during or after the generation of the polyploid. We report here studies of NOR number,

*Current address and correspondence: Department of Botany, University of Reading, Whiteknights, Reading RG6 2AS, UK.
NOR activity and ribosomal cistron locations in the polyploid complex of Scilla autumnalis which shows such a reduction in NOR numbers associated with ploidy.

Scilla autumnalis is a plant of the family Liliaceae widespread in Europe, North Africa and western Asia, which shows remarkable chromosomal diversity (Ainsworth et al., 1983; Parker et al., 1991). Within this single morphological species, ten cytologically distinct races have been detected comprising five distinct diploids as well as auto- and allopolyploids. In this paper the NORs of two of the genomes designated $A$ and $B^{7}$, both comprising seven chromosomes, have been examined using standard Feulgen staining to detect secondary constrictions, silver staining to establish NOR expression, in situ hybridization to localize the chromosomal sites of ribosomal cistrons and $\mathrm{C}$-banding and fluorochrome banding to define heterochromatic blocks associated with NORs.

\section{Materials and methods}

Plants of genomic constitution $A A(2 n=14)$ and $A A B^{7} B^{7}(2 n=28)$ were collected from natural populations in Spain and Portugal. $B^{7} B^{7}$ diploids $(2 n=14)$ were obtained from Corfu, Greece. $B^{7} B^{7} B^{7} B^{7}(2 n=28)$ plants were collected in South Devon, England and $A A B^{7} B^{7} B^{7} B^{7}(2 n=42)$ plants from the Lizard Peninsula, Cornwall. 
These plants were crossed to produce offspring with the genome constitutions shown in Table 1. Cytological preparations were made from root tips pretreated with 0.05 per cent colchicine for $3 \mathrm{~h}$, fixed overnight in $3: 1$ ethanol:acetic acid at room temperature and stored in fixative at $-20^{\circ} \mathrm{C}$.

Standard staining was carried out by the Feulgen method. C-banding, silver nitrate staining and fluorochrome staining techniques were modified from Schwarzacher et al. (1980), Kodama et al. (1980) and Schweizer (1976, 1980), respectively. In situ hybridization of the wheat ribosomal probe pTA71 (Gerlach \& Bedbrook, 1979) was carried out by the method of Clark et al. (1989).

\section{Results}

\section{Number of secondary constrictions in the chromosome complements}

The $A A$ and $B^{7} B^{7}$ genomes are morphologically similar although the $A$ genome contains 70 per cent more DNA than the $B^{7}$ genome (Ainsworth et al., 1983). The two genomes can be unequivocally distinguished on the basis of size and the chromosomes

Table 1 Parent and offspring genomes

Parents

\begin{tabular}{ll}
\hline \multicolumn{1}{c}{$\sigma^{7}$} & Offspring \\
\hline$B^{7} B^{7} B^{7} B^{7} \times B^{7} B^{7}$ & $B^{7} B^{7} B^{7}$ \\
$A A B^{7} B^{7} \times B^{7} B^{7}$ & $A B^{7} B^{7}$ \\
$A A \times B^{7} B^{7}$ & $A A B^{7}$ \\
$A A B^{7} B^{7} \times B^{7} B^{7} B^{7} B^{7}$ & $A B^{7} B^{7} B^{7}$ \\
$A A B^{7} B^{7} B^{7} B^{7} \times B^{7} B^{7} B^{7} B^{7}$ & $A B^{7} B^{7} B^{7} B^{7}$ \\
$A A B^{7} B^{7} B^{7} B^{7} \times A B^{7} B^{7}$ & $A A B^{7} B^{7} B^{7}$ \\
\hline
\end{tabular}

within each complement can be individually recognized. Each complement has a single chromosome carrying a NOR visible as a secondary constriction adjacent to the centromere in the long arm (Fig. 1). These chromosomes are referred to as $A 3$ and $B^{7} 3$, respectively.

In the autotetraploid $B^{7} B^{7} B^{7} B^{7}$, four secondary constrictions are normally visible in Feulgen-stained complements, with three in synthetic triploids produced by $B^{7} B^{7} B^{7} B^{7} \times B^{7} B^{7}$ hybridization. In the allopolyploids of constitutions $A A B^{7} B^{7}$ and $A A B^{7} B^{7} B^{7} B^{7}$, however, the $A 3$ chromosomes show no secondary constrictions. The chromosome complements of these races thus contain two and four secondary constrictions, respectively, identifiable as those on the $B^{7} 3$ chromosomes (Fig. 1).

The number of chromosomes with secondary constrictions in inter-racial crosses involving polyploids conforms to the values predicted from the parental numbers and thus corresponds to the number of $B^{7}$ genomes present. For example, the tetraploid of constitution $A B^{7} B^{7} B^{7}$ has three NO-chromosomes as does the pentaploid $A A B^{7} B^{7} B^{7}$ and all of these are represented by $B^{7} 3$.

The triploid hybrid $A A B^{7}$, obtained from a diploid $\times$ diploid cross after non-disjunction in the $A A$ egg parent, has NORs on both $A 3$ chromosomes and the single $B^{7} 3$. Thus there is no suppression of the activity of $A 3$ by $B 3$ chromosomes, at least in the ratio of $2: 1$ found in this plant.

\section{Nucleolar-organizer expression}

The number of active NORs detected by silver staining was in all cases identical to the number of secondary constrictions visualized in Feulgen-stained cytological preparations (Fig. 2). Thus, the secondary constriction of the $B^{7}$-genome chromosome 3 was shown to be site of nucleolar-organizing activity in all genetic constitutions and ploidy levels (Figs 3 and 4). In the $A$ genome,

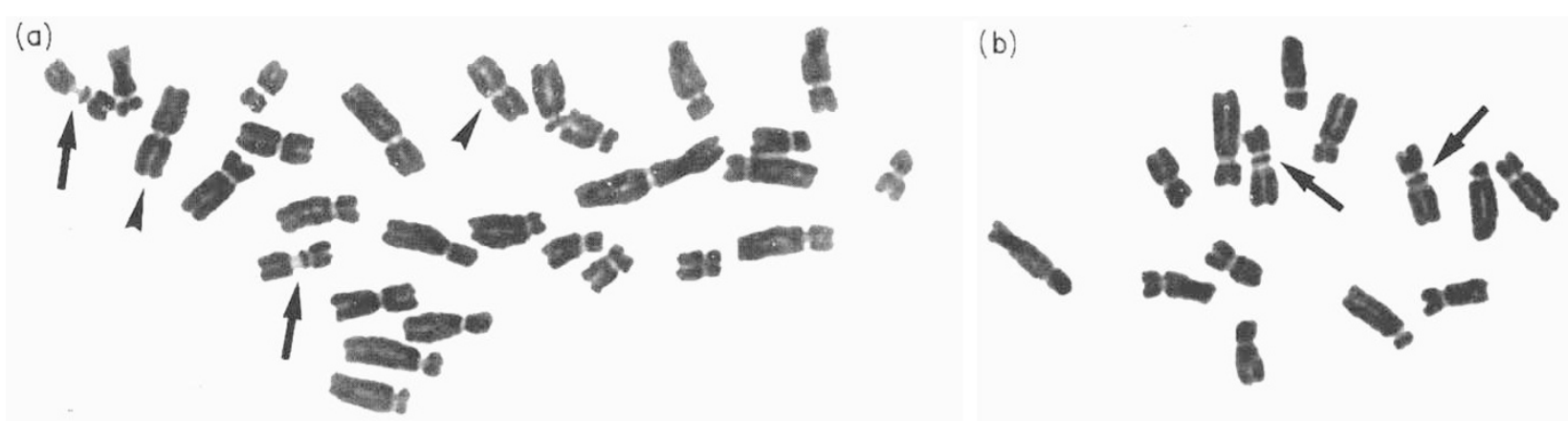

Fig. 1 Chromosome complements of (a) $A A B^{7} B^{7}$ and (b) $A A$ plants of Scilla autumnalis. Two nucleolar-organizer regions in each complement (long arrows) and deleted chromosome A 3 in the tetraploid (arrow heads) are shown. 

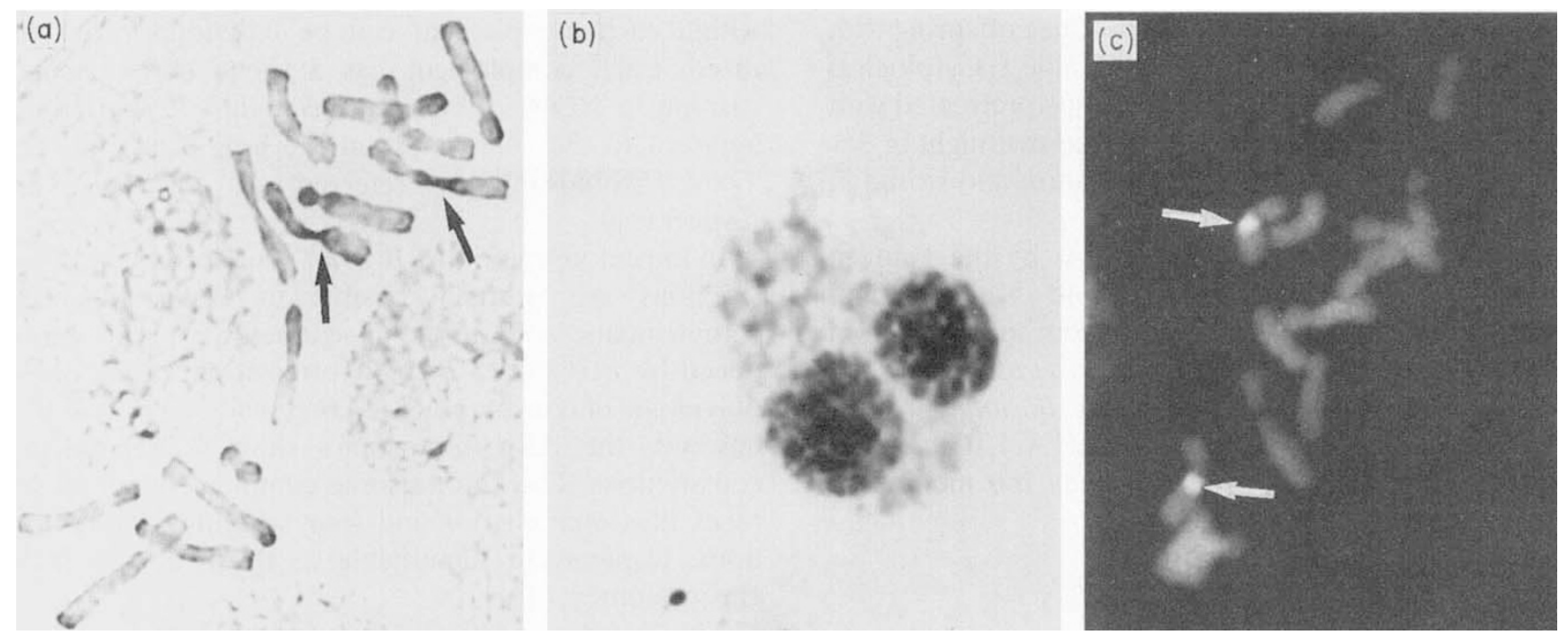

Fig. 2 AA diploid: (a) silver staining, (b) silver-stained nucleus with two nucleoli, (c) fluorescent banding with $\mathrm{CMA}_{3}$. Arrows indicate NORs.
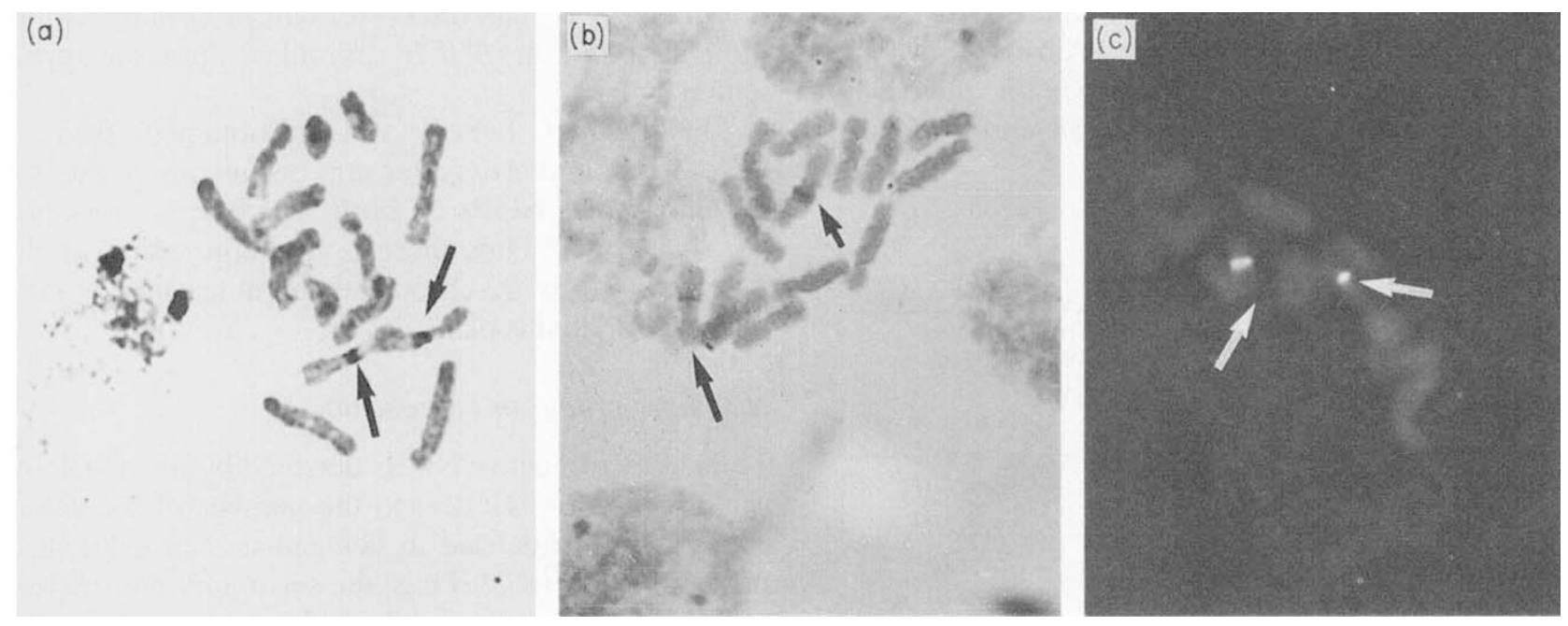

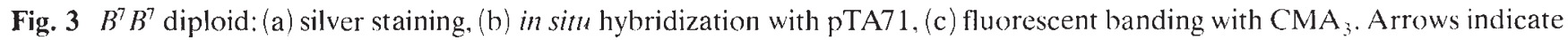
NORs.

NOR activity was detected only at the diploid level and in the $A A B^{7}$ triploid offspring derived from the diploid parent.

The silver-stained region of chromosome $A 3$ was generally larger than that of $B^{7} 3$, and this was especially noticeable in the $A A B^{7}$ triploid which carried two large and one small silver-stained region in all cells. The amounts of deposited silver at the secondary constrictions varied between chromosomes and between individuals in the different ploidy levels and this phenotype was consistent, reproducible and transmissable.
The maximum number of nucleoli observed in nuclei after silver-staining corresponded closely to the number of secondary constrictions seen in Feulgenstained complements and the number of sites of chromosomal silver deposition.

\section{C-banding and $\mathrm{CMA}_{3}$ fluorescence patterns}

Only one C-band is observed in the $A$ and $B^{7}$ genomes of diploid $S$. autumnalis and this is at the site of the NOR on $A 3$ and $B^{7} 3$, respectively (Lozano et al., 1990; Vaughan, 1990). This heterochromatic band is 


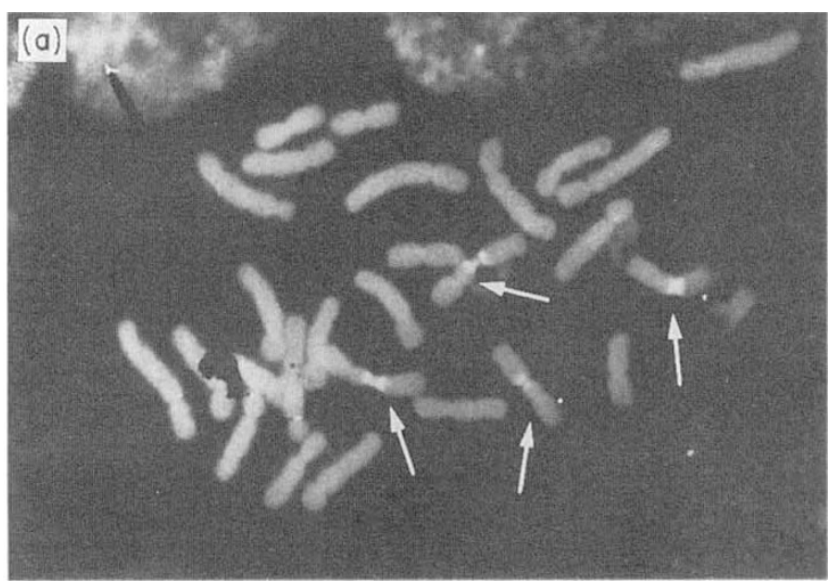

(b)

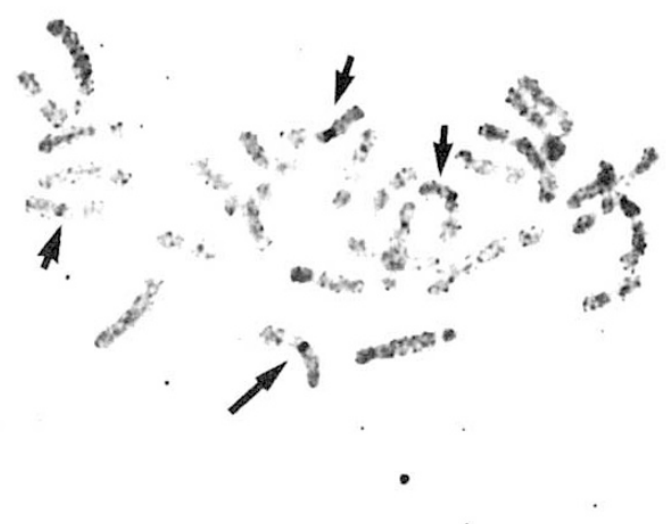

Fig. $4 B^{7} B^{7} B^{7} B^{7}$ tetraploid with four NORs: (a) silver staining, (b) fluorescent banding with $\mathrm{CMA}_{3}$. Four organizers arrowed.

$\mathrm{CMA}_{3}$ positive and DAPI negative indicating that it is GC-rich (Figs 2-4). This C-band is entirely digested by treatment with the restriction enzyme HaellI (Lozano et al., 1990).

In the $A$ genomes of the tetraploid race $A A B^{7} B^{7}$ and the hexaploid race $A A B^{7} B^{7} B^{7} B^{7}$ no $\mathrm{C}$-bands or $\mathrm{CMA}_{3}$-bright bands could be detected (Figs 5 and 6 ). They were also absent from $A 3$ chromosomes in all four hybrids derived from these races: $A B^{7} B^{7}$, $A B^{7} B^{7} B^{7}, \quad A B^{7} B^{7} B^{7} B^{7}$ and $A A B^{7} B^{7} B^{7}$. The $B^{7} 3$ chromosomes, however, maintained the expected C-banding and fluorescent phenotype.

\section{In situ hybridization}

In situ hybridization to the metaphase chromosomes of S. autumnalis was carried out using the ribosomal gene probe pTA71 which contains a $9 \mathrm{~kb}$ insert of a wheat ribosomal cistron (Gerlach \& Bedbrook, 1979). The probe was labelled with biotin by nick translation and detection employed streptavidin/horseradish peroxidase.

In metaphase complements the sites of hybridization of pTA71 corresponded to the regions of nucleolarorganizer activity detected by silver staining. Thus the diploids $A A$ and $B^{7} B^{7}$ always exhibited two regions of hybridization at the positions of the NORs on chromosomes $A 3$ and $B^{7} 3$. The $A 3$ chromosome in races at the polyploid level, $A A B^{7} B^{7}, A A B^{7} B^{7} B^{7} B^{7}$ and their derivatives, showed no sites of hybridization. Again, in the polyploids the numbers of sites corresponded to the numbers of copies of the $B^{7} 3$ chromosomes in the complement (Figs 3, 5 and 6).

The amount of deposition at the hybridizing sites on the chromosomes often varies. In the $A A B^{7}$ triploid carrying three organizer regions the sites on the two
A3 chromosomes were consistently larger than the $B^{7} 3$ site. Although the detection system is not quantitative, the pattern of size variation observed after in situ hybridization corresponds to that seen after silver staining, indicating that the activity of the NORs may be a direct reflection of ribosomal cistron number.

\section{Discussion}

The application of silver-staining, C-banding, fluorescence banding and in situ hybridization techniques to the chromosome complements of diploid, allotetraploid and autoallohexaploid races of Scilla autumnalis has demonstrated that during, or subsequent to, polyploidization the nucleolar-organizing capacity of the $A$ genome chromosomes has been eliminated. After in situ hybridization, no signal was visible on chromosome $A 3$ in the polyploid, so the rDNA is either entirely absent or the numbers of copies of the ribosomal cistrons are so low that they are below the level of detection by this method. Similarly, no silver-binding could be seen on $A 3$ and no micronucleoli were observed in interphase nuclei. The NOR region in $A A$ and $B^{7} B^{7}$ diploids and in $B^{7} 3$ chromosomes in tetraploids has a $\mathrm{CMA}_{3}$-positive $\mathrm{C}$-band adjacent to it but this band was absent from chromosome $A 3$ of allotetraploids. Thus, elimination of nucleolar-organizer activity has involved deletion both of the ribosomal cistrons themselves and the adjacent heterochromatin.

Very few cases of ribosomal cistron elimination in polyploids have been demonstrated. The clearest examples are in species of the wheat group. At the diploid level, most species including Triticum monococcum with genome constitution AA have two pairs of chromosomes with ribosomal cistron sites. Aegilops squarrosa, the D genome donor of hexaploid bread 

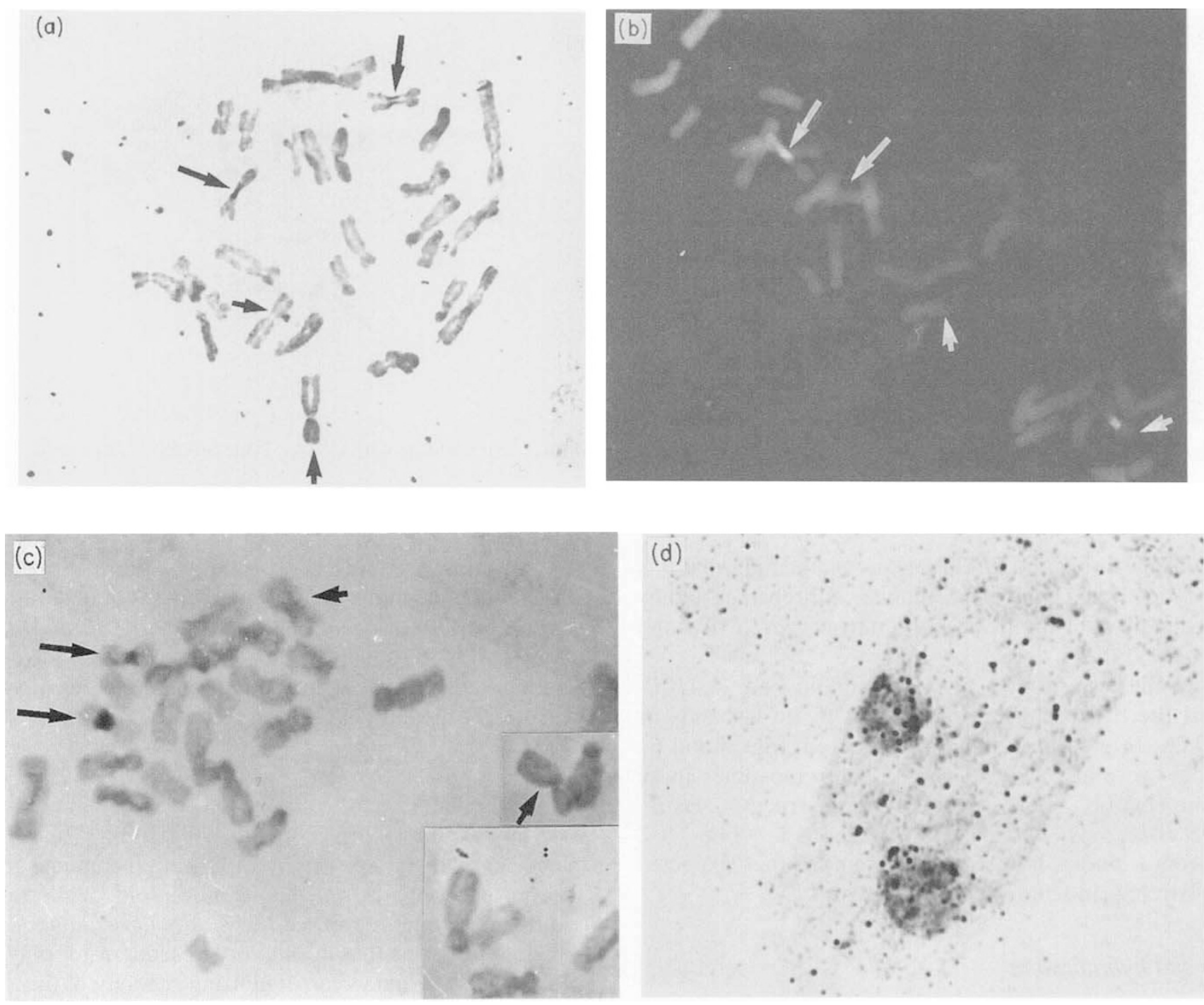

Fig. 5 NORs in $A A B^{7} B^{7}$ tetraploids: (a) silver staining, (b) fluorescent banding with $\mathrm{CMA}_{3}$, (c) in situ hybridization with pTA71, (d) silver-stained nucleus with two nucleoli. $B^{7} 3$ chromosomes (long arrows) and the $A 3$ chromosomes (arrow heads) are seen. Note the absence of staining and hybridization to $A 3$ chromosomes.

wheat, is exceptional as it carries only a single pair (Gerlach et al., 1980; Teoh et al., 1983). The tetraploids of constitution $\mathrm{AAB}^{7} \mathrm{~B}^{7}$ such as $T$. dicoccum also have two pairs of sites and these are referable to the satellited chromosomes $1 \mathrm{~B}$ and $6 \mathrm{~B}$. The NOR regions of the two pairs derived from the $A$ genome have thus been eliminated (Hutchinson \& Miller, 1982). The hexaploid wheats, similarly have reduced numbers of rRNA loci with only two in the variety 'Chinese Spring' but four in Triticum spelta (Miller et al., 1980). Control of nucleolar-organizer expression in the wheat group, however, is complex: in polyploid species of the genus Aegilops the number of rRNA loci is consistently higher than the number of satellite chromosome pairs (Teoh et al., 1983) indicating the frequent occurrence of nucleolar dominance (amphiplasty) (Martini et al., 1982; Lacadena et al., 1988). In Triticum aestivum, when a dominant nucleolar-organizer is removed, recessive organizers become more active in compensation (Martini \& Flavell, 1985).

In polyploids, it is frequently assumed that an observed reduction in the number of NORs from that expected from summing the numbers in the diploid progenitors is a result of nucleolar suppression. In the tetraploid species of Avena, for example, one or two pairs of organizers are absent from the $\mathrm{AABB}$ and AACC tetraploids (Fominaya et al., 1988). On the basis of silver-staining, which only detects activelyexpressed nucleolar-organizer regions, Fominaya et al. (1988) concluded that the reduced numbers in polyploids are the result of nucleolar competition. As indicated from the results obtained in this study of Scilla, 

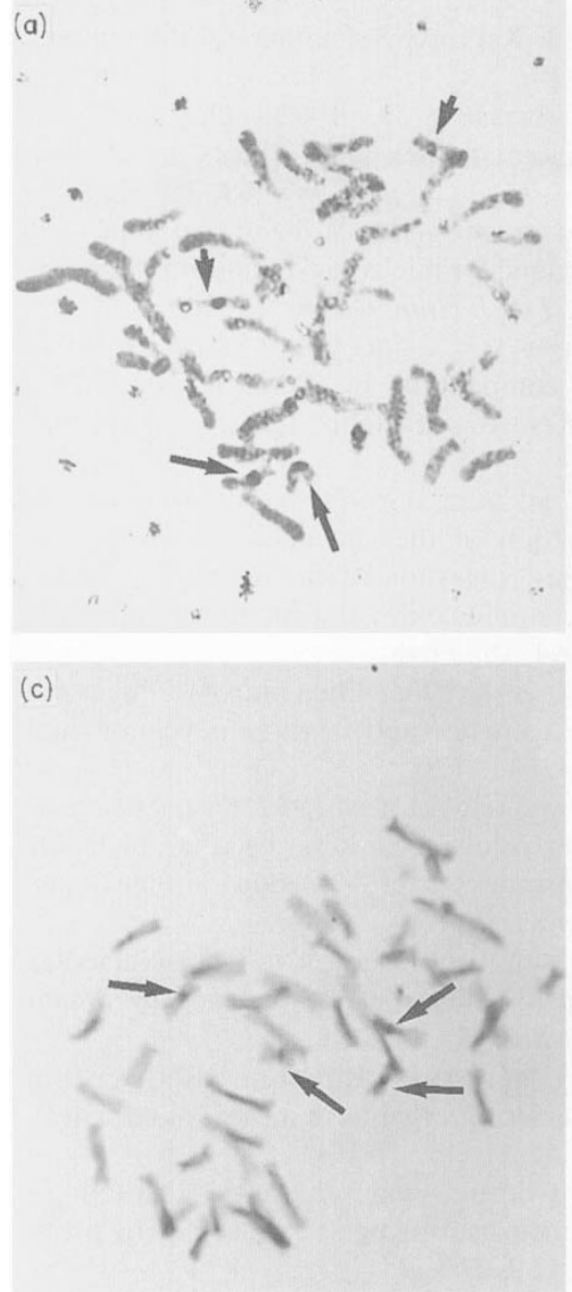
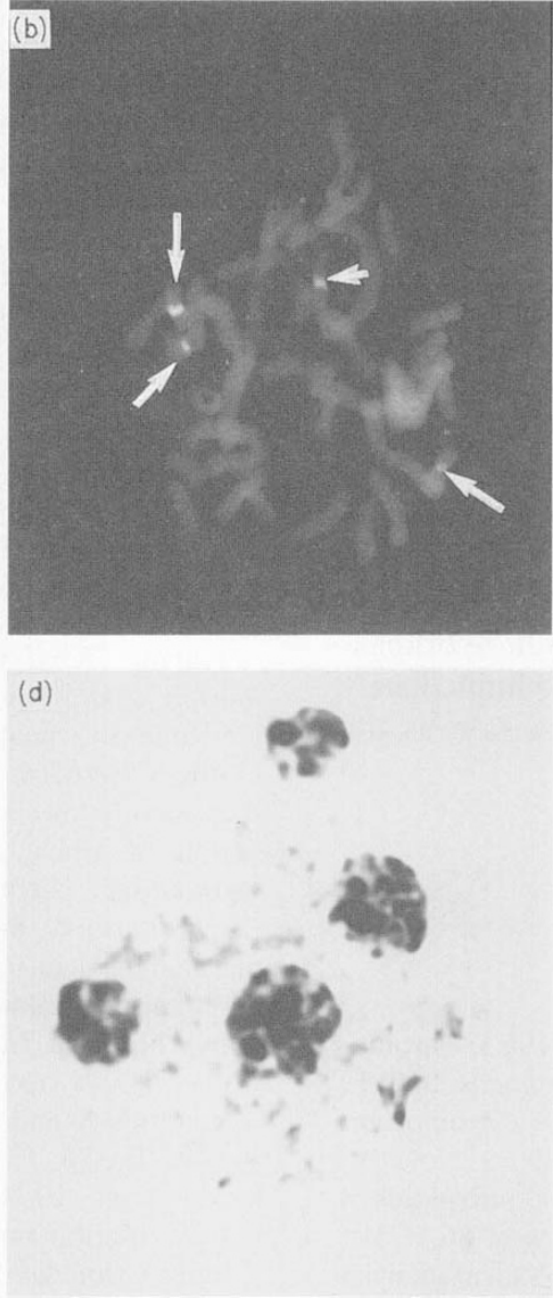

Fig. 6 NORs in $A A B^{7} B^{7} B^{7} B^{7}$ hexaploids: (a) silver staining, (b) fluorescent banding with $\mathrm{CMA}_{3}$, (c) in situ hybridization with pTA71, (d) silver-stained nucleus with four nucleoli. Four $B^{7} 3$ chromosomes are seen (arrows). evidence from in situ hybridization with rDNA probes is required before this conclusion can be reached.

The number of sites of rDNA in the complement can be very variable. In Allium schoenoprasum, for example, most populations carry a nucleolar-organizer region only on the short arm of chromosome 8 (Bougourd \& Parker, 1976). In some populations, however, an additional site occurs on the long arm of chromosome 7 (M. Garrido-Ramos et al., unpublished data). Such variation in NOR number has been attributed to different processes, such as deletions or transpositions (Schubert \& Wobus, 1985), but the direction of change either towards an increase or decrease in the number of sites is usually not easy to determine. In $A$. schoenoprasum, Garrido-Ramos et al. concluded that deletion of sites had occurred during the evolution of this species. Clear evidence of NOR deletion has been found in Triticum aestivum. A wheat aneuploid stock tetrasomic for chromosome 5D had 40 per cent fewer ribosomal cistrons than standard individuals of the variety 'Chinese Spring' (Mohan \& Flavell, 1974). In situ hybridization of the wheat ribosomal probe pTA71 to this stock confirmed that one of the major nucleolus organizers, either on chromosome $1 \mathrm{~B}$ or $6 \mathrm{~B}$, had been entirely eliminated (Miller et al., 1980). These two organizers account for about 70 per cent of the total ribosomal cistrons present in hexaploid bread wheat.

A remarkably similar series of observations to those reported here in $S$. autumnalis has been made in the polyploid complex of the East Asian species Scilla scilloides. This species has a diploid race with $2 n=16$ containing genome $\mathrm{A}$ and another diploid with $2 n=18$ carrying a distinctive genome B (Haga \& Noda, 1976). Secondary constrictions are found on chromosomes a2 in genome $\mathrm{A}$ and $\mathrm{b} 1$ in genome $\mathrm{B}$. In the $\mathrm{F}_{1}$ hybrid $\mathrm{AB}$, $2 n=17$, both $\mathrm{a} 2$ and $\mathrm{b} 1$ chromosomes are present, while the allotetraploid $\mathrm{AAB}^{7} \mathrm{~B}^{7}$ with $2 n=34$ carries only a pair of b1 chromosomes. On the basis of this evidence Araki (1985) deduced that a diploid carrying a variant form of the A genome, lacking a secondary constriction, was the progenitor of the allotetraploid. As this individual would lack nucleolar-organizer 
activity and be unable to synthesize rRNA it is an unlikely hypothesis. It would seem more likely that deletion of the a2 NOR occurred coincident with or subsequent to polyploidization as in $S$. autumnalis. It cannot be excluded in this case, however, that the A genome donor to the allotetraploid carried ribosomal cistron variants which exhibited nucleolar-suppression in combination with the $\mathrm{B}$ genome organizer. The differential activity of nucleolar-organizers can be ascribed to the organization of the non-transcribed spacer region in terms of the number and structure of repeat units (A repeats) or the methylation status of specific cytosines (Flavell, 1989; Flavell et al., 1986). Variation in A repeat numbers from 6 to 17 has been found in wild tetraploid wheats from Israel (Flavell et al., 1986). In tetraploid S. scilloides, in situ hybridization with a ribosomal cistron probe would immediately distinguish between amphiplasty and excision as the basis of the NOR reduction.

\section{References}

AINSWORTHI, C. C., PARKER. J. S. AND HORTON, D. M. 1983. Chromosome variation and evolution in Scilla autumnalis. In: P. E. Brandham and M. D. Bennett (eds). Kew Chromosome Conference II.pp. 261-268.

ARAKI, H. 1985. The distribution of diploids and polyploids of the Scilla scilloides complex in Korea. Genetica, 66, 3-10.

BENNETT, S. T. AND THOMAS, S. M. 1991. Karyological analysis and genome size in Milimm (Gramineae) with special reference to polyploidy and chromosomal evolution. Genome, 34. 868-878.

BOUgOURD, S. M. AND PARKER, J. S. 1976. Nucleolar-organiser polymorphism in natural populations of Allium schoenoprasum. Chromosoma, 56, 301-307.

ClARK, M. S., KARP, A. ANI ARCHER, S. A. 1989. Physical mapping of $\mathrm{B}$ hordein loci on chromosome 5 by in situ hybridisation. Genome, 32, 925-929.

FLAVELL., R. B. 1989. Variation in structure and expression of ribosomal DNA loci in wheat. Genome, 31. 963-968.

FI.AVELL, R. B., O'DELL. M., THOMPSON, W. F., WINCENTZ, M., SARDANA, R. AND BARKER. R. F. 1986. The differential expression of ribosomal RNA genes. Phil. Trans. R. Soc. Lond. B, 314. 385-397.

FOMINAYA, A., VEgA, C. AND FERRER, E. 1988. C-banding and nucleolar-activity of tetraploid Avena species. Genome. 30. 633-638.

GERLACI, W. L. AND BEDBROOK, J. R. 1979. Cloning and characterisation of ribosomal RNA genes from wheat and barley. Nuclei Acid Res., 1, 1865-1885.

GERLACH, W. L., MILler, T. E. AND FlaVELl, R. B. 1980. The nucleolus organisers of diploid wheats revealed by in situ hybridisation. Theor. Appl. Genet., 58, 97-100.
HAGA, T. AND NODA, S. 1976. Cytogenetics of the Scilla scilloides complex. 1. Karyotype genome and population. Genetica, 46, 161-176.

HUTCHINSON, J. AND MILLER, T. E. 1982. The nucleolar organisers of tetraploid and hexaploid wheats revealed by in situ hybridisation. Theor. Appl. Genet., 61, 285-288.

KODAMA. Y., YOSHIDA, M. C. AND SASAKI, M. 1980. An improved silver staining technique for nucleolus organiser regions by using a nylon cloth. Jpn. J. Hum. Genet., 25, 229-233.

I.ACADENA, J. R., CERMEÑO, M. C., ORELLANA, I. AND SANTOS, J. L. 1988. Nucleolar competition in Triticeae. In: P. E. Brandham (ed.), Kew Chromosome Conference III, pp. $151-165$.

LOZANO, R., JAMILENA, M., RUIZ REJON, C. AND RUIZ REJON, M. 1990. Characterisation of the chromatin of some liliaceous species after digestion with restriction endonucleases and sequential Giemsa, fluorochrome and silver staining. Heredity, 64. 185-195.

MARTINI. G. AND FLAVELL, R. B. 1985. The control of nucleolus volume in wheat: a genetic study at three developmental stages. Heredity, 54, 111-120.

MARTINI, G., ODELL, M. AND Fl.AVFl.L, R. B. 1982. Partial inactivation of wheat nucleolus organisers by the nucleolus organiser chromosomes from Aegilops umbellulata. Chromosoma, 84, 687-700).

MILL.ER. T. E., GERL_ACH, W. L. AND Fl.AVELl, R. B. 1980. Nucleolus organiser variation in wheat and rye revealed by in situ hybridisation. Heredity, 45. 377-382.

MOHAN, J. AND FLAVELL, R. B. 1974. Ribosomal RNA cistron multiplicity and nucleolar organisers in hexaploid wheat. Genetics, 76. 33-34.

NAVASHIN, M. 1934. Chromosomal alterations caused by hybridisation and their bearing upon certain genetic problems. Cytologia, 5, 169-203.

PARKER, J. S., LOZANO. R., TAYLOR, S. AND RUIZ REJON, M. 1991. Chromosomal structure of populations of Scilla autumnalis in the Jberian Peninsula. Heredity, 67, 287-297.

SCHUBERT, I. AND WOBIS, U. 1985. In sith hybridisation confirms jumping nucleolus organiser regions in Allitm. Chromosoma, 92, 143-148.

SChwarzacher, T.. Ambros, P. AND schweizer. D. 1980. Application of Giemsa banding to orchid karyotype analysis. Pl. Syst. Evol., 134, 293-297.

SCHWEIZER, D. 1976. Reverse fluorescent chromosome banding with chromomycin and DAPI. Chromosoma, 58. 307-324.

SCHWEIZER, D. 1980. Simultaneous fluorescent staining of R-bands and specific heterochromatic regions (DA/DAPI bands) in human chromosomes. Cytogenet. Cell Genet. 27. 190-193.

TEOH. S. B.. HUTCHINSON, J. AND MILLER, T. E. 1983. A comparison of the chromosomal distribution of cloned repetitive DNA sequences in different Aegilops species. Heredity, 51 , 635-641.

VAUGHAN, H. E. 1990. Chromosomal and genetical analysis of the Scilla autumnalis complex. Ph.D. Thesis, University of London. 